\title{
on the prevention of lightning
}

\begin{abstract}
The destructive nature of cloud-to-ground lightning strokes is well known. Loss of life and damage to buildings and other man-made structures may to a large extent be prevented by the judicial use of lightning conductors and screens but no comparable protection may be offered to expanses of agricultural crops or forests. According to Fuquay (1967) lightning is the greatest single cause of forest fires in the western United States: during the period 1946-1962, 140,000 such fires occurred causing severe losses of timber, wildlife, watershed, and recreational resources. Comparab!e losses occur regularly in other parts of the world. The only solution is the suppression or modification of cloud-to-ground lightning discharges. Methods of suppression are described, some of which may turn out to be practical ways of achieving this aim.
\end{abstract}

\section{The thundercloud and the lightning stroke}

Lightning is only observed to occur in clouds of vigorous convective activity, containing strong vertical air currents whose velocity may exceed $30 \mathrm{~m} \mathrm{sec}^{-1}$, although values of $5-10 \mathrm{~m} \mathrm{sec}^{-1}$ are more common. The storm is generally composed of "cells" of 1-2 km horizontal extent and depth (from cloud base to cloud top) typically about $7 \mathrm{~km}$. The bases of thunderstorms are usually near the $0 \mathrm{C}$ isotherm, though the exact temperature level varies with the geographical location, being as warm as $+10 \mathrm{C}$ or even $+15 \mathrm{C}$ over the mid-western plains and on occasion below $0 \mathrm{C}$ in northern and more mountainous regions.

Electrically, the cloud exhibits a dipole structure with positive charge uppermost and a region of mixed charges in the center. Fig. 1 shows that the bulk of the cloud is more or less electrically neutral. This model is a somewhat simplified one but is sufficient for the present discussion. When the total potential difference between the charge centers is of the order $10^{8}-10^{9}$ vo!ts dielectric breakdown will occur within the cloud and an intra-cloud flash takes place. Similarly wherever the electric field strength below the cloud becomes very large a cloud-to-ground flash will ensue. During a flash about 100 coulomb-kilometers of electric moment is destroyed and about 25 coulombs of charge transferred in a period of time of about $100 \mathrm{msec}$, usually bringing negative charge to Earth. Brook et al. (1962a) and Brook et al. (1962b) have made a quantitative analysis of about 200 electrical and photographic records of cloud-to-ground discharges and have concluded that flashes may be divided into single and multiple discrete flashes, and single and multiple (hybrid) continuing flashes. They observed that $90 \%$ of cloud-to-ground flashes were multiple flashes and that, of these, $50 \%$ involved one or more continuing components.

The continuing component is of long duration and low current, perhaps 200 amperes for 200 milliseconds. Brook et al. showed that a discrete stroke, whether part of a discrete flash or a hybrid flash, was associated with an average change in electric moment of $20 \mathrm{ckm}$, whilst a continuing current interval was associated with an average value of $135 \mathrm{ckm}$. Fuquay et al. (1967) consider from observations made on seven lightning discharges which started fires in Montana, that, since all of them contained continuing-current phases, this is the primary means by which lightning fires are caused.

One of the ways of reducing the incidence of fires caused by lightning may therefore be to modify in some way the discharge mechanism so that continuing components are less likely to occur. The means by which this might be effected is not at present known and further investigation of the conditions which lead to the

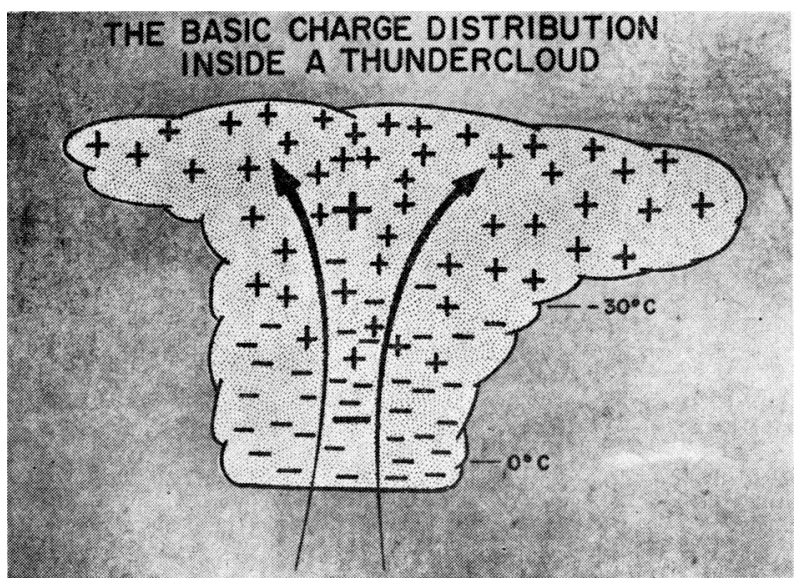

Fig. 1. Simplified model of a charge distribution within a large cumulus cloud. 
occurrence of continuing currents is needed. At the moment the suppression of the lightning flash seems to be a much more realizable proposition.

\section{Lightning suppression}

Since lightning is associated with convective clouds, that is, clouds containing vigorous updrafts and downdrafts of large vertical extent, the most direct method of suppression would be to eliminate the clouds themselves or else convection within them. The elimination of entire cloud systems entails the removal of moisture from the atmosphere by some means and also the removal of temperature instability. Such large-scale modifications are not feasible technologically, economically or energetically. The reduction of convection is, however, possible.

Convective clouds form when moist air is forced upwards either by thermal instability or by orographic influences and cools adiabatically to produce water. The condensation releases heat which causes further buoyancy and further entrainment of moist air from below. If the water undergoes a further transition and freezes then the latent heat so released enables the air supporting the cloud particles to rise to even higher altitudes. Since the natural nucleation temperature within a cloud is usually about $-15 \mathrm{C}$ or colder the increase in buoyancy of an air parcel when freezing takes place can be considerable. The time at which nucleation takes place during the development of a convective cloud is extremely critical in determining its eventual size and activity. Glaciation which occurs as a consequence of nuclei naturally present in the cloud proceeds as a continuous process, beginning slowly at first and increasing as the air parcel cools; in these circumstances the cloud is usually well organized and preparing to enter the mature stage.

Artificial nucleation by seeding may reinforce natural glaciation and assist the cloud in its development. On the other hand rapid, premature seeding, while increasing buoyancy, may also give rise to a sufficiently high degree of turbulence that the development of the cloud is inhibited. The colder air entering the cloud by a turbulent mixing process will substantially lower the chances of the cloud penetrating or even reaching inversion layers at moderate altitudes. Late nucleation, on the other hand, may be ineffective if the cloud is already entering its dissipative stage.

Assuming no specific theory for the mechanism of charge generation, but bearing in mind the very high correlation between the height of the cloud, convective activity and lightning activity, then the early nucleation of a cloud should reduce the probability of intense electrical activity. In addition the reduction in the vertical extent of the cloud should make intra-cloud discharges more probable than cloud-to-ground discharges. It is worth noting that many fires initiated by lightning strokes are subsequently extinguished by precipitation. Over enthusiastic cloud seeding frequently produces a "dry" cloud (when the cloud is entirely above the freezing isotherm) which produces negligible or no precipitation. Fuquay (1967) has shown that such clouds occur naturally and frequently, for instance, in Montana, where forest fires are particularly common. Clearly the advantage of reduced cloud-to-ground stroke activity must be weighed carefully against the possible reduction in precipitation.

The seeding is effected by releasing silver iodide into the cores of growing cumulus clouds and may be introduced from ground level or from aircraft flying in the updrafts beneath cloud base. It is important to release the nucleating agent as near to the cloud as possible as silver iodide is rapidly deactivated by exposure to light. The effectiveness of the seeding is measured partly from observations and photographs of cloud growth and partly from a thorough probing of the cloud at various times and altitudes after seeding using an instrumented aircraft. The principal measurement is that of the size and number distribution of cloud particles and hydrometeors. The cloud particles are examined using a replication technique devised by Schaefer (1956) and developed by Meteorology Research Incorporated. The cloud particles are examined by exposing a continuously moving strip of $16-\mathrm{mm}$ polyester film coated with ethylene formvar to the airstream and then drying the exposed film thereby leaving a permanent plastic replica of the previously embedded ice crystals or droplets. Fig. 2 shows examples of the records obtained in this way. Hydrometeors (generally cloud particles of diameter greater than $1000 \mu$ ) were examined by the exposure to the airstream of a continuously moving strip of soft metal foil. Particles of hydrometeor size leave impressions characteristic of their size and constitution enabling water drops, graupel, hail and ice crystals to be readily distinguished. Fig. 3 shows a typical record of cloud hydrometeors. Using both techniques simultaneously it is possible to estimate what fraction of the material constituting a cloud is glaciated. Fig. 4 shows typical values of the time required after seeding to achieve a given degree of glaciation.

It should be stated here that the amount of material used to initiate glaciation must be chosen carefully. Too little seeding materials may have no appreciable effect upon the clouds dynamical process. Moderate amounts of the material may even enhance the growth of a cumulus cloud. Effective dissipation is best achieved by over-seeding though, as pointed out previously, the lack of precipitation may defeat the objective if the prevention of fires is the prime concern. Against this must be balanced the cost of using excessive amounts of a seeding agent; silver iodide, for example, is a relatively expensive chemical.

From the point of view of the dynamical processes, therefore, the cloud may most likely be rendered inactive by the copious, early seeding of its central updraft region using an aircraft flying below cloud base. Mixing within the cloud will rapidly distribute the nucleating agent to the majority of the cloud volume. 

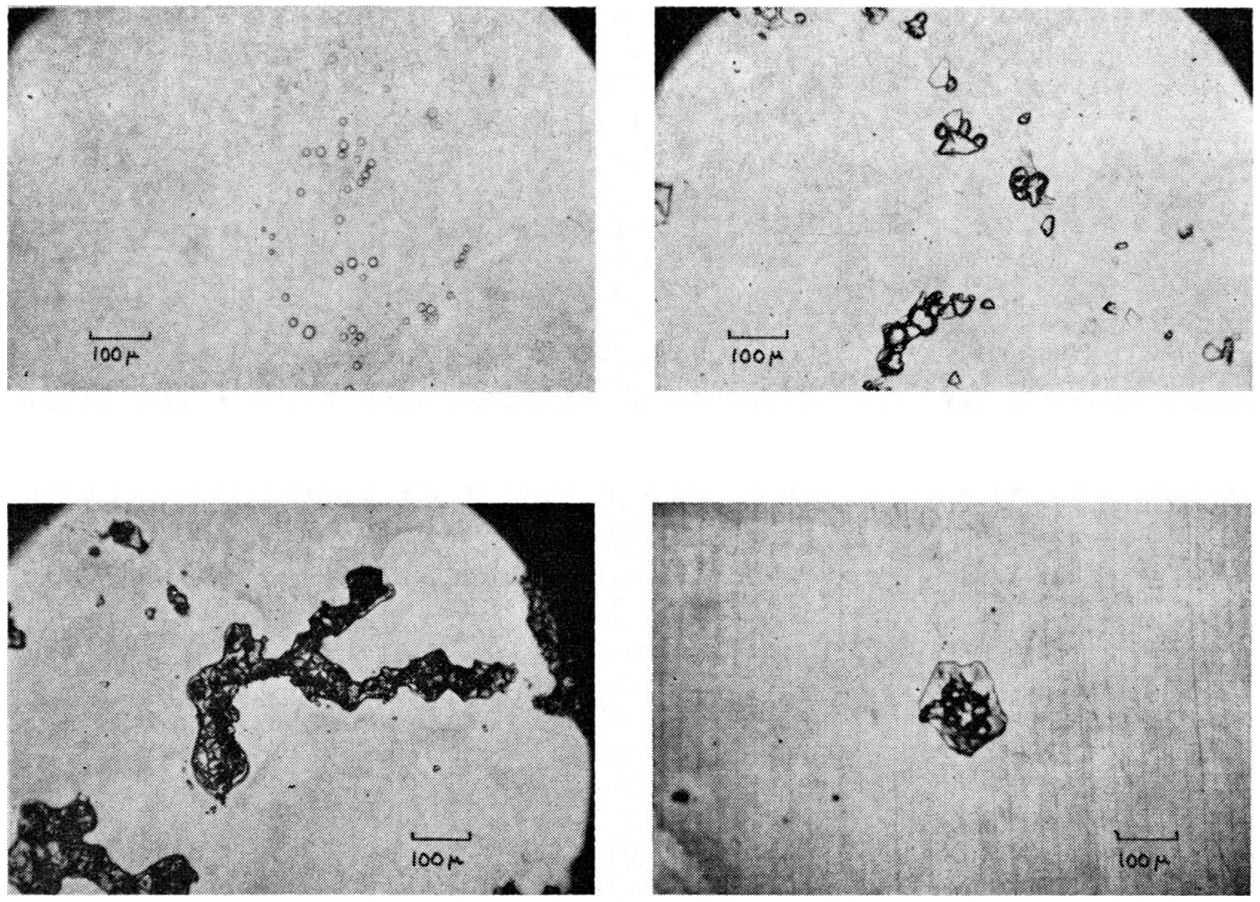

Fig. 2. Records of cloud particles obtained on ethylene formvar showing droplets (top left), ice crystals (top right), rimed aggregates (bottom left), and a cluster of frozen droplets on which ice has grown from the vapor.

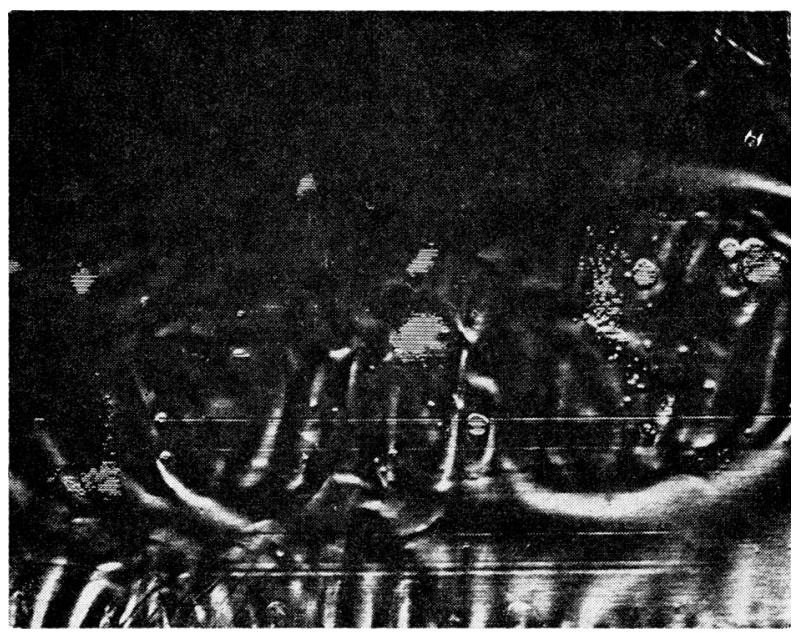

FIG. 3. Hydrometeor records obtained on soft metal foil showing graupel, shattered pellets and drops. The parallel reference lines on the indentations are $100 \mu$ apart.

\section{An alternative approach}

So far we have not been concerned with the exact nature of the charge-generation mechanisms within thunderclouds. A more efficient method of lightning suppression may be to interfere with the conditions which encourage charge separation by means of the mechanisms. Considerable effort has been put into examining the physical and electrical behavior of convective clouds and a number of theories are currently purporting to

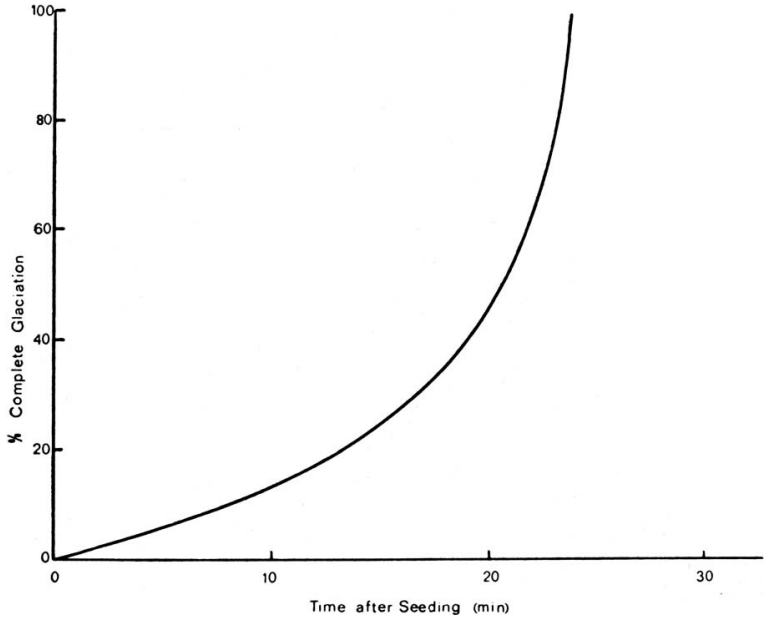

FIG. 4. The rate of glaciation of cumulus clouds using a silver iodide seeding agent distributed immediately below cloud base from an aircraft.

explain the production of lightning. In order to evaluate these theories it is necessary to measure simultaneously within a cloud variables such as hydrometeor charge, liquid water content, electric field strength, temperature, the distribution and nature of cloud particles and hydrometeors and the wind field (updrafts and downdrafts).

During the summers of 1966 and 1967 Latham and Stow made intensive studies of convective clouds at 
Flagstaff, Arizona. The objective of the investigation was to determine the structure of cumulus clouds at various stages of their development and to correlate, if possible, the physical and electrical properties of each cloud. The measurement of electric field strength was effected by using a rotating cylindrical field mill of the type devised by Kasemir (1964). The instrument was mounted on the nose of a Piper Aztec aircraft with its axis parallel to that of the aircraft. In addition to measuring the vertical field strength when in horizontal flight it also measures the strength of the electric field in a horizontal direction perpendicular to the line of flight. Correct positioning of the instrument minimized the contribution to these fields caused by self-charging of the aircraft. It was possible to measure fields ranging from about 1 volt per centimeter to breakdown magnitudes to within $10 \%$.

The measurement of hydrometeor charge was made using a development of the induction technique devised originally by Gunn (1947). The device consisted of two electrically screened and insulated cylinders mounted under the nose of the aircraft with their axes almost parallel to the longitudinal axis of the airplane. Charged hydrometeors which passed through the device caused a charge of related magnitude to be induced on the cylinders in turn, thereby producing two pulses on an oscillograph. Pairs of output signals which correspond in magnitude and sign were taken as meaningful and others rejected. Using this device it was possible to measure to within an accuracy of $10 \%$ charges ranging in magnitude from about $10^{-2}$ to 1.0 esu.

Measurements of updrafts and downdrafts, liquidwater content and temperature were made using standard techniques. All measurements other than particle size and type were recorded simultaneously on the oscillograph.

After climbing to its maximum altitude the aircraft made spiral descents through a chosen cloud, or, alternatively, traversed the cloud horizontally at a series of temperature-levels down to just below cloud base. Where possible airborne measurements were supplemented with ground-based radar information, timelapse cloud photographs and meteorological soundings. The majority of studies were made on natural clouds though some clouds had been artificially glaciated by introducing silver iodide particles.

\section{The theories of cloud electrification}

a) Convective charging. Vonnegut (1955) proposed a mechanism for the formation of thunderstorm electricity which does not involve precipitation elements. He proposed that the electrification in thunderstorms is a result of strong updrafts and downdrafts that cause an increase of the small space charge normally found in the lower atmosphere. Because the air in the lower atmosphere contains a small positive space charge, clouds formed of this air have a small positive electric charge and produce an electric field. Under the influ- ence of this field negative ions from the upper atmosphere move down toward the cloud. These ions do not neutralize the cloud because they are caught in downdrafts that carry them down to the lower part of the cloud where they accumulate to form a large region of negative space charge. This charge finally becomes large enough to produce a large positive space charge by corona from points on the ground beneath the cloud. Air containing this increased positive space charge is carried by updrafts to the top of the cloud, causing it to become even more positively charged. This increases the rate of growth of the center of negative charge and finally the charges become large enough to produce lightning.

b) Freezing potentials. Workman and Reynolds (1948, 1950) showed that when water freezes an electric potential is developed across the ice/water interface. The electrification is thought to be a consequence of the selective incorporation of impurity ions into the solid during freezing and is a sensitive function of the kind and amount of impurity added to the water. It has been proposed that cloud microphysical processes that involve freezing and splashing may result in the separation of charge, the collision of supercooled water drops and hailstones, for example, causing the splashed droplets to carry away an opposite sign of charge to that conferred on the hailstone.

c) Riming and collision. Reynolds et al. (1957) describe some important experiments which they performed in order to study the electrification of an artificial hailstone during riming. When the hailstone was exposed to an airstream containing a mixture of supercooled droplets and ice crystals it became charged appreciably. Negligible charging occurred when the cloud was composed only of supercooled water or of ice crystals. Reynolds et al. believed the charging to be caused by temperature differences between the hailstone and the impacting ice crystals, the riming process serving to warm the surface of the hailstone by the release of latent heat. Within a cloud gravitational separation of charge would then take place.

d) Riming and splintering. Latham and Mason (1961) performed a series of experiments to investigate the separation of electric charge caused by the collision of supercooled water droplets of uniform size with an artificial hailstone. It was observed that the hailstone became charged when the accretion and freezing of droplets upon it was accompanied by the production and ejection of ice splinters. Splinter production was observed to be a function of impact velocity and drop diameter. Since the splinters, which carry an opposite sign of charge to that on the hailstone, are generally very much smaller than the hailstone, gravitational separation of charge occurs.

e) Inductive charging. If sets of particles of different sizes are in free fall within a cloud then one set will interpenetrate the other by virtue of the difference in fall speeds. If the particles are polarized by a weak vertical electric field (usually present in the atmosphere) 
then collisions not involving coalescence will result in the net separation of charge between the sets. The separation of charge will be in such a direction as to reinforce the prevailing electric field and further collisions will separate greater amounts of electric charge. Müller-Hillebrand (1954) has proposed a mechanism for inductive charging which involves only the ice phase; a more general analysis of inductive charge separation has been given by Sartor $(1960,1961 \mathrm{a}, 1961 \mathrm{~b}, 1965)$ which may be applied to the much more complex problem of drop-droplet interactions.

All of the above mechanisms, represented diagrammatically in Fig. 5, are, in theory, capable of providing charging at a rate sufficient to produce lightning. In order to suppress lightning, or at least appreciably diminish the frequency of occurrence of lightning strokes, it is clearly necessary to know which of the mechanisms is primarily responsible for cloud electrification under a given set of meteorological conditions.

Some of the more important conclusions of the aircraft probing experiments may be deduced from Fig. 6 . The figure shows the records obtained during one of several horizontal traverses when the aircraft penetrated two adjacent clouds of similar exterior dimensions; the parameters measured were liquid water content, temperature, hydrometeor charge, and the concentrations of supercooled water drops, ice crystals and graupel pellets. It may be seen that the interior of the clouds exhibit quite different properties, the Cloud (1) to the left of the figure containing only a few ice crystals and a large number of supercooled water drops while the Cloud (2) to the right of the figure contains a considerable number of ice crystals and graupel pellets and some supercooled water; the temperature measured during the traverse remained appreciably constant at approximately -10C. No hydrometeor charges were measured in Cloud (1) and the electric fields were of the order of the external fair-weather value of $100 \mathrm{Vm}^{-1}$ : yet in Cloud (2) appreciable charging was observed and vertical electric fields were frequently greater than $10,000 \mathrm{Vm}^{-1}$. It was noted that the electrification of the cloud was strongest at points where ice crystals, supercooled droplets and graupel pellets co-existed and that electrification was appreciably diminished at points where either the crystals, droplets or graupel pellets were absent.

Since the convective activity of the two clouds was observed to be of the same magnitude (as demonstrated by cloud-top heights and the motion of the aircraft) it may be inferred that convective activity alone was not responsible for the electrification of Cloud (2) and that the convective mechanism of Vonnegut was not the principal generator of the electric fields measured. Furthermore, the riming and splintering process of Latham and Mason would appear to have been ineffective in separating charge since Latham and Mason found in their experiments that inappreciable quantities of charge were separated when the cloud droplet diameter was less than $30 \mu$. In the measurements made using the
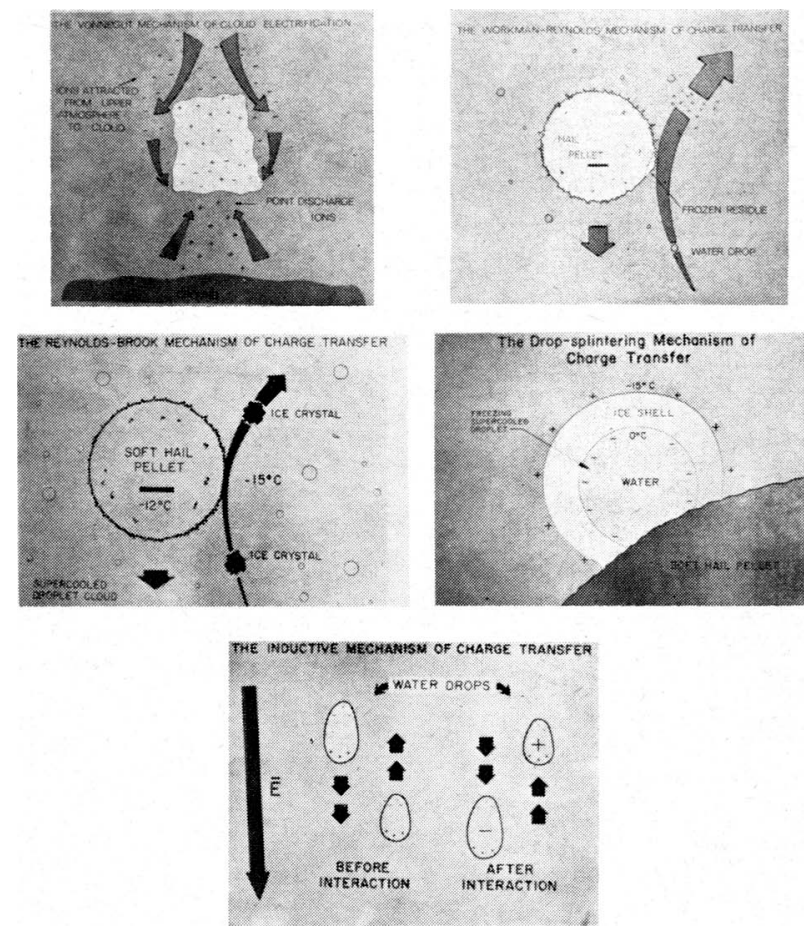

FIG. 5. Schematic illustrations of the five principal theories of thunderstorm electrification described in the text.

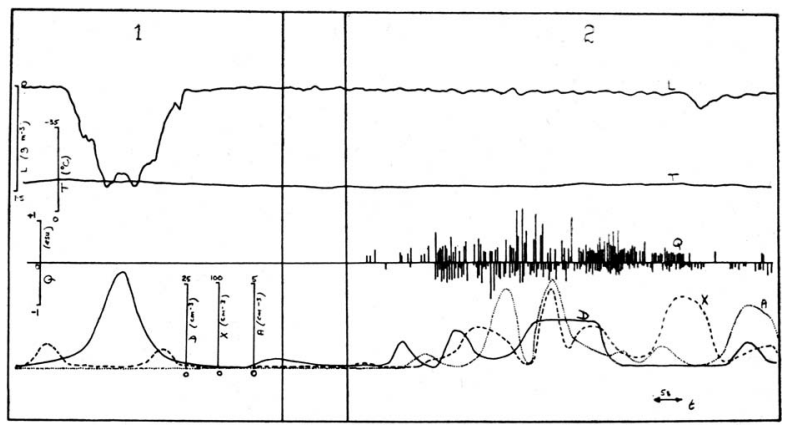

Fig. 6. Oscillographic records of liquid-water content L, temperature $T$, hydometeor charge $Q$, compared with the computed population densities of supercooled water droplets $\mathrm{D}$, small ice crystals $\mathrm{X}$, and rimed aggregates $\mathrm{A}$. The records show fundamental differences between cloud 1 , which is barely glaciated, and cloud 2, which is appreciably glaciated, at the $-10 \mathrm{C}$ isotherm.

aircraft very few cloud droplets were observed which had diameters in excess of $30 \mu$ and therefore the number of effective charge-generating events must have been extremely small.

Evidence for and against the possibility that charging was effected by the Workman-Reynolds freezing process is scanty, largely because the mechanism by which charge might be separated in a given cloud is speculative and hard to assess. Indeed, the most substantial argument against the Workman-Reynolds process is the negative one that almost all the evidence obtained from the airborne measurements support the process suggested and 
investigated by Reynolds, Brook and Gourley. The requirement laid down by Reynolds et al. that charging should be appreciable was that hailstones should exist in an environment containing large numbers of both ice crystals and supercooled water drops. It was only under these conditions that appreciable electrification was observed inside the clouds studied.

Evidence for the inductive process proposed by Müller-Hillebrand was afforded by one well documented occasion when the polarity of charge on the hydrometeors and the direction of the vertical electric field strength vector were reversed. Convective activity was not great and no water, supercooled or otherwise, was present in the cloud. It therefore appears likely that the inductive mechanism made some contribution to the electrification of the majority of the clouds studied.

Since the physical constitution of a convective cloud is dictated by a large number of meteorological and geographical factors the conclusion that charging is a consequence primarily of the Reynolds, Brook and Gourley process with some contributions from an inductive mechanism must be restricted to clouds occurring during the summer in northern Arizona. Similar measurements made in Montana during late summer by the author have shown that the process of Reynolds et al. could not have been responsible since the clouds were completely glaciated. In such clouds electrification must have been a consequence of convective charging or collisions between ice particles involving melting and refreezing, transfer of polarization charges, or transfer of charges caused by temperature differences between the particles. Insufficient data is yet available to determine which of these mechanisms is dominant.

It seems unlikely, therefore, that one specific method of treatment could be applied to convective clouds in order to prohibit or at least reduce lightning activity, though the possibility remains that a given treatment may be capable of suppressing more than one charge generating process.

\section{Suppression methods}

a) Convective charging. The charging process is initiated by the convection of space charge gathering beneath a forming cumulus cloud and a possible method of modifying the process is to introduce space charge of the opposite polarity to that naturally occurring under the cloud. Vonnegut and Moore (1959), Semonin et al. (1962) and Vonnegut et al. (1962) attempted to influence, with success, the electrification of cumulus clouds by the deliberate introduction of space charge. The space charge was generated beneath the clouds by corona discharge from a high-tension wire, the ions so produced becoming attached to condensation nuclei, and introduced into the cloud by natural updrafts. Their results showed that the electrical state of a developing cumulus cloud was affected by the space charge, the cloud acquiring a charge of the same sign as that introduced. They also showed that an opposite sign of space charge was present around the edges of the cloud. It is not yet clear just how significant electrification of this type may be in determining the electrical status of the cloud when it becomes mature. Even if space-charge suppression were effective the engineering problems of implementing it over any reasonable area would be of the highest magnitude.

b) Workman-Reynolds freezing process. According to Workman (1967) the constitution of any water found in clouds is such as to ensure strong electrification with the cloud dipole having net positive charge situated above net negative charge (which polarity is almost invariably observed in clouds); the polarity is determined by the concentration and type of ionic impurities in the water forming the cloud. Of the commonly available substances those containing the ammonium radical are found to cause electrical charge separation during freezing opposite in polarity to that associated with rain water. Therefore the introduction by rockets, aircraft or ground generators of salts containing the ammonium radical into cloud droplets would be expected to inhibit or reverse the charging which is a consequence of the freezing process.

c) Reynolds, Brook and Gourley ice crystal-hailstone collision. According to the experiments of Reynolds et al. the charges generated by the collisions of ice crystals with hailstones are considerably reduced when supercooled droplets are not also present. This finding is supported by the airborne measurements described earlier in this article, though the electrification of completely glaciated clouds is not necessarily insufficient to cause lightning in some circumstances.

Supercooled droplets may be removed from a convective cloud by over-seeding with ice-forming nuclei.

d) Latham-Mason drop splinter mechanism. The 30diameter limit below which supercooled drops will not shatter has not been unequivocally established. Since this criterion is the only one which might exclude the mechanism from being considered important it is worth considering how the separation of charge might be prevented.

Again, the most obvious way is to remove supercooled water from the cloud, causing it to become completely glaciated by the introduction of freezing agents. The mechanism is expected to be sensitive to impurities in the water, though the way in which charge separation is affected by the addition of impurities has not been explicitly studied.

e) Inductive charging. The efficiency of the inductive charging process increases as the number of collisions between particles increases, as the interpenetration (gravitational separation) increases, and as the probability of separation after collision (no coalescence) increases.

Since the coalescence efficiency of water droplets is greater than that of ice crystals there would appear to be an argument for limiting the degree of glaciation within a cloud in order to keep inductive charging to a minimum. However, the presence of water in a developing cumulus cloud almost always leads to particle 
growth by coalescence or freezing and this in turn leads to gravitational separation of particles and an enhancement of inductive charging. Interpenetration of cloud particles is minimized if the dispersion of particle sizes is minimized. The stabilization of the particle-size spectrum may be achieved by the rapid freezing of droplets before they have a chance to grow by coalescence or other means. So yet again it would appear that glaciation by over-seeding is the solution to the problem, provided that it is done during the early stages of cloud development.

Thus there appear to be three distinct approaches to lightning suppression. Seeding with nucleating agents will both inhibit convective activity and prevent certain charge generating processes from occurring. The injection of space charge may nullify convective charging. The introduction of the ammonium radical may affect electrification caused by the freezing of water. In a given cloud system it is unlikely that one mechanism will be solely responsible for the electrification and it may not, therefore, be necessary to quench all the generating processes at one time in order to reduce charging to below the lightning threshold. Cloud seeding would appear to be the most powerful method of modifying the cloud's physical processes provided the seeding can be done at the right moment. The quenching of a mature cloud system by seeding may be impossible; on the other hand, a mature system will almost certainly precipitate and may thus alleviate the risk of fire. Such lightning suppression methods are at present entirely speculative and even if they are effective, as may be proved by further airborne investigations, considerations of economy may exclude their use.

Acknowledgments. This author wishes to express his gratitude to Meteorology Research Incorporated, Altadena, Calif., for providing data (Fig. 4) and also the facilities which made the airborne electrification studies possible. $\mathrm{He}$ also wishes to thank the National Science Foundation of the United States for financial support, and the Forest Fire Service, Missoula, Mont., for their cooperation.

\section{References}

Brook, M., N. Kitagawa and E. J. Workman, 1962a: Continuing currents in cloud-to-ground lightning discharges. $J$. Geophys. Res., 67, 637-647.

$\longrightarrow,-$ and $\longrightarrow, 1962 \mathrm{~b}$ : Quantitative study of strokes and continuing currents in lightning discharges to ground. J. Geophys. Res., 67, 649-659.
Fuquay, D. M., 1967: Weather modification and forest fires. Amer. Assoc. Adv. Sci., 309-325.

—, R. G. Baugman, A. R. Taylor and R. G. Hawe, 1967: Characteristics of seven lightning discharges that caused forest fires. J. Geophys. Res., 72, 6371-6373.

Gunn, R., 1947: The electrical charge on precipitation at various altitudes and its relation to thunderstorms. Phys. Rev., 71, 181-186.

Kasemir, H., 1964: The cylindrical field mill. Tech. Rep. ECOM-2526, U. S. Army Electronics Command, Fort Monmouth, New Jersey, U. S. A.

Latham, J., and B. J. Mason, 1961: The generation of electric charge associated with the formation of soft hail in thunderclouds. Proc. Roy. Soc., A260, 537.

Müller-Hillebrand, D., 1954: Charge generation in thunderstorms by collision of ice crystals with graupel, falling through a vertical electric field. Tellus, 6, 367-381.

Reynolds, S. E., M. Brook and M. F. Gourley, 1957: Thunderstorm charge separation. J. Meteor., 14, 4260436.

Sartor, J. D., 1960: Some electrostatic cloud-droplet collision efficiencies. J. Geophys. Res., 65, 1953-1957.

—, 1961a: Calculations of cloud electrification based on a general charge-separation mechanism. J. Geophys. Res., 66, 831-838.

- 1 1961b: Recalculations of cloud electrification based on a general charge-separating mechanism. J. Geophys. Res., 66, 3070-3071.

-, 1965: Induction charging thunderstorm mechanism. Problems of Space and Atmos. Elect., Elsevier Press, 307310.

Semonin, R. G., D. W. Staggs and G. E. Stout, 1962: Cloud electrification studies in Illinois. Ann. Rep. AD283-453, NSF-G-17083.

Schaefer, V. J., 1956: The preparation of snow crystal replicas. Weatherwise, 9, 132-135.

Vonnegut, B., 1955: Possible mechanism for the formation of thunderstorm electricity. Geophys. Res. Paper 42, Air Force Cambridge Res. Center, Mass., 169-181.

- , and C. B. Moore, 1959: Preliminary attempts to influence convective electrification in cumulus clouds by the introduction of space charge into the lower atmosphere. Recent Advances in Atmos. Elect., Pergamon Press, 317.

$\longrightarrow,-$, R. G. Semonin, J. W. Bullock, D. W. Staggs and W. C. Bradley, 1962: Effect of atmospheric space charge on initial electrification of cumulus clouds. J. Geophys. Res., 67, 3909-3922.

Workman, E. J., 1967: The production of thunderstorm electricity. J. Franklin Inst., 283, 540-557.

- , and S. E. Reynolds, 1948: A suggested mechanism for the generation of thunderstorm electricity. Phys. Rev., 74, 709 .

—, and - 1950: Electrical phenomena occurring during the freezing of dilute aqueous solution and their possible relationship to thunderstorm electricity. Phys. Rev., 78, 254-259. 


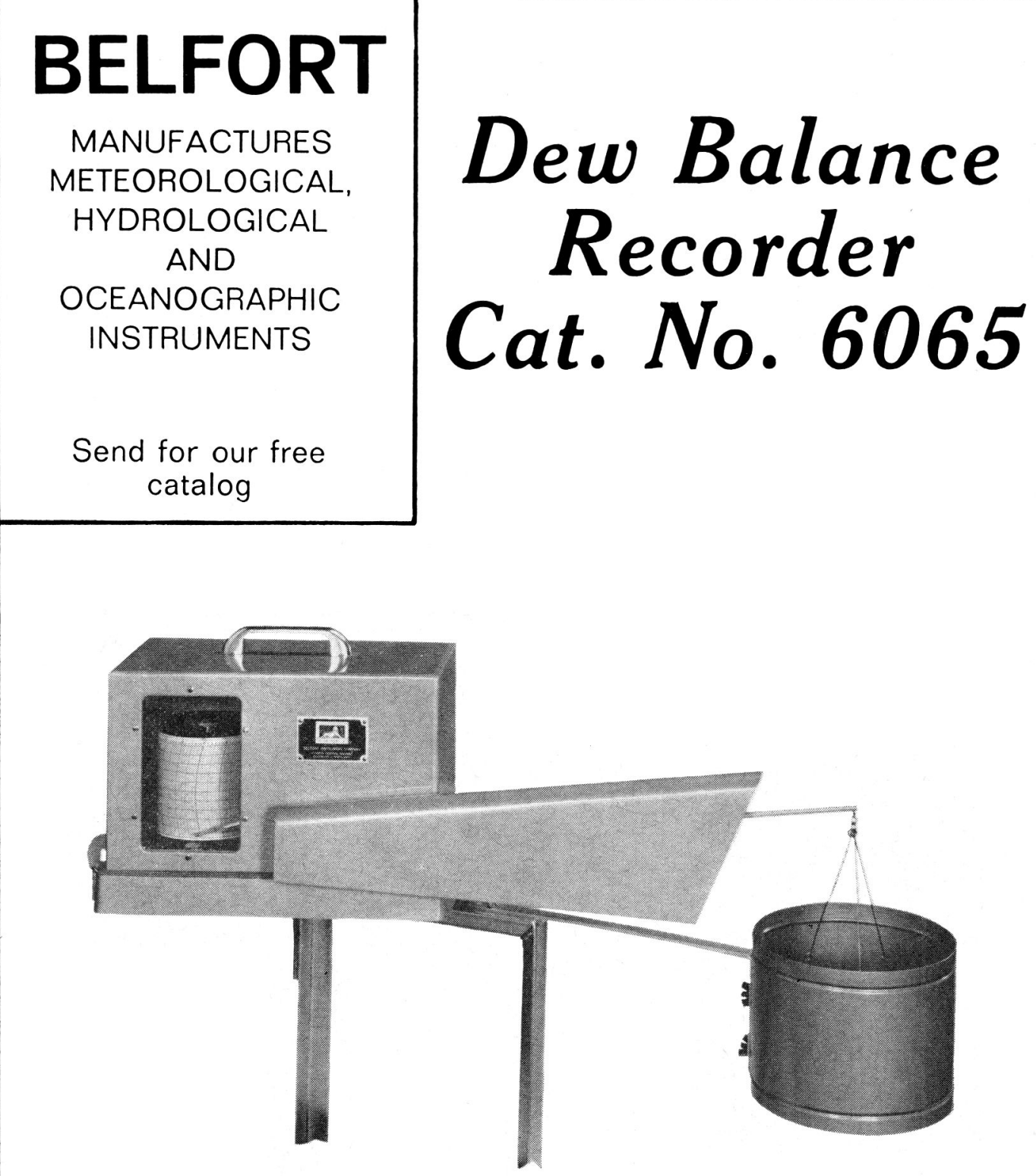

SENSITIVITY: 0.05 GRAMS

RANGE: 0 to 5.0 GRAMS

CHART DURATION: 7 DAYS

NOTE:

OUR NEW ADDRESS 1600 SOUTH CLINTON STREET

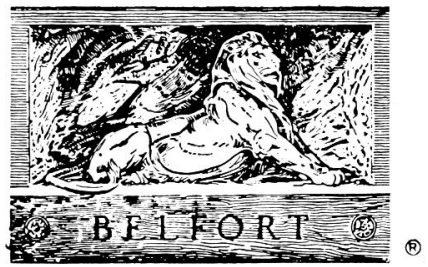

\section{BELFORT INSTRUMENT COMPANY}

1600 S. CLINTON STREET

BALTIMORE, MARYLAND 21224 U.S.A. 